\title{
Petite fabrique de la connaissance : aborder le savoir scientifique en se racontant des histoires
}

\section{Alain Chartier et Catherine Frier}

\section{(2) OpenEdition}

1 Journals

\section{Édition électronique}

URL : https://journals.openedition.org/pratiques/1439

DOI : $10.4000 /$ pratiques. 1439

ISSN : 2425-2042

Éditeur

Centre de recherche sur les médiations (CREM)

\section{Édition imprimée}

Date de publication : 15 décembre 2009

Pagination : 168-186

\section{Référence électronique}

Alain Chartier et Catherine Frier, « Petite fabrique de la connaissance : aborder le savoir scientifique en se racontant des histoires », Pratiques [En ligne], 143-144 | 2009, mis en ligne le 19 juin 2014, consulté le 21 septembre 2021. URL : http://journals.openedition.org/pratiques/1439 ; DOI : https://doi.org/ 10.4000/pratiques. 1439

\section{(c) Tous droits réservés}




\title{
Petite fabrique de la connaissance : aborder le savoir scientifique en se racontant des histoires
}

\author{
Alain Chartier \\ IUFM Grenoble, Collège de la Motte d'Aveillans \\ Catherine Frier
}

Université Stendhal, Lidilem

\section{Introduction}

Ce texte évalue les effets d'un dispositif expérimenté depuis deux ans dans le cadre d'un cours dispensé par l'UFR de Sciences du Langage à l'université Stendhal et visant l'appropriation de savoirs théoriques par le biais de l'activité scripturale (atelier de lecture/écriture). Le travail proposé amène les étudiants à construire des connaissances théoriques dans leur domaine disciplinaire dans et par l'écrit, la finalisation du travail prenant la forme originale d'une fiction scientifique, réalisée dans le cadre d'un atelier d'écriture. Cette démarche se fonde sur l'hypothèse suivante : pour s'approprier un savoir il faut l'apprivoiser, et pour cela le faire entrer en résonance avec sa propre histoire. L'écrit produit dans le cadre du dispositif est par conséquent susceptible de favoriser cette articulation entre « singulier» (pôle de la subjectivité) et "générique » (pôle de l'objectivité scientifique), en reliant des espaces habituellement coupés de façon hermétique dans le cadre des apprentissages scolaires. L'évaluation de la démarche permet d'explorer des pistes didactiques inédites, de révéler et de mettre en valeur des compétences scripturales souvent ignorées chez les étudiants.

\section{Quelques jalons théoriques}

"Il y aurait donc place, croyons-nous, pour une psychanalyse indirecte et seconde, qui chercherait toujours l'inconscient sous le conscient, la valeur subjective sous l'évidence objective, la rêverie sous l'expérience. On ne peut étudier que ce qu'on a d'abord rêvé. La science se forme plutôt sur une rêverie que sur une expérience et il faut bien des expériences pour effacer la brume du songe » (Gaston Bachelard, La psychanalyse du feu, 1949, réédition 1986 : 23-44).

Notre article trouve sa source dans le champ des recherches sur les pratiques de 
lecture/écriture comme modes de construction des savoirs dans l'enseignement supérieur. Ce champ est loin d'être nouveau en didactique et a déjà donné lieu à de très nombreuses publications ${ }^{(1)}$ dans des perspectives pluridisciplinaires. Ces travaux mettent souvent l'accent sur les difficultés rencontrées par les jeunes étudiants lors de leur première rencontre avec les textes de spécialité dans leur discipline (Frier, 1998). Difficulté à aborder la « connaissance théorique », à intégrer et à restituer notions et concepts de façon précise, à problématiser, à objectiver le savoir, à insérer le discours d'autrui dans son propre discours (Kara, 2004), à prendre en charge un point de vue (Rinck, 2004), à articuler théorie et expériences de terrain (Frier, 2004). Ces travaux insistent également sur la fonction heuristique de l'écrit et sur la dimension réflexive des pratiques scripturales qui favorisent la construction des connaissances et la « codification scripturale des savoirs » propre à la « raison graphique ». Depuis quelques décennies seulement, un autre pan de la didactique s'intéresse plus spécifiquement au rôle joué par le récit dans la construction de la connaissance en général et du savoir scientifique en particulier, autrement dit à la fonction didactique du récit dans les différentes disciplines (Reuter, 2007). Cette tendance est assez nouvelle puisque jusqu'à une époque récente la place de la narration dans la formation scientifique était très controversée : «très généralement, on trouve une méfiance vis-à-vis de la narration considérée comme antinomique du discours scientifique » (Bautier et al, 2000:158). Traditionnellement en effet on considère que la démarche scientifique implique l'effacement du locuteur et de sa subjectivité au profit d'une objectivation de l'expérience, le dépassement du local et du contingent au profit de la généralisation et de la systématisation des faits. Et dans cette optique la « raison graphique» est perçue comme le meilleur outil pour mettre en œuvre cette classification systématique du réel qui permet l'abstraction, la distanciation, l'objectivité scientifique. La polémique suscitée par la mise en place de l'épreuve d'écriture d'invention au baccalauréat de français et le rejet qu'elle a suscité au départ (Petitjean, 2005 : 76), illustrent parfaitement cette réticence première, ce déni de « sérieux » à l'égard de l'univers de la fiction qui n'aurait rien en commun avec celui du savoir et de la raison.

Pourtant nombreux sont les chercheurs de différents horizons qui remettent aujourd'hui en question cette vision positiviste un peu restrictive du savoir scientifique et de sa construction en défendant une « heuristique narrative » (Bautier, 2000 : 159) et en redonnant une place au récit dans l'enseignement scientifique. Jérôme Bruner fut l'un des premiers à mettre en avant l'enjeu cognitif du récit en démontrant la dimension intellectuelle du mode narratif et le lien fort, anthropologique, entre récit, pensée et culture au sens large. Selon lui notre esprit connaît deux modes de fonctionnement, chacun pouvant être traduit dans l'autre : le mode paradigmatique, assertif, qui rend compte du fonctionnement des choses, et le mode narratif, qui lui s'intéresse plutôt à ce que les choses auraient pu être ou à ce qu'elle pourraient être : "nous vivons simultanément dans ces deux modes : celui, austère mais bien délimité, du mode de pensée paradigmatique, et celui, plein de défis, du récit. C'est en réalité lorsque nous perdons de vue que les deux modes de pensée sont liés l'un à l'autre que nous cessons d'être à même de faire face aux évènements » (Bruner, 2005 : 123).

(1) Voir notamment le numéro 121-122 de Pratiques (2004) : les Ecrits Universitaires, coordonné par F. Boch, I. Laborde-Milaa, Y. Reuter, qui constitue une très bonne synthèse sur la question. 
Afin d'aider nos étudiants à aborder la connaissance scientifique et de leur permettre de «faire face aux évènements », nous avons nous aussi décidé de prendre au sérieux les histoires que nous nous racontons. Il y a plusieurs raisons à cela. Tout d'abord parce que nous nous situons dans une perspective constructiviste de l'apprentissage qui considère que celui qui apprend ne peut s'approprier le savoir de façon passive, mécanique. Autrement dit pour que la connaissance spontanée croise le savoir scientifique, il faut que se creuse l'espace d'une rencontre, que se construise une histoire commune. D'autre part parce que nous nous situons dans une conception didactique centrée sur l'apprenant, qui fait une place au sujet et à sa singularité (Delamotte et al., $2000: 3$ ). C'est pourquoi nous pensons qu' "apprendre c'est créer ce que seul le sujet apprenant peut faire pour lui-même : un réseau de relations actives entre ce qu'il sait et ce qu'il assimile. L'apprenant ne réinvente pas l'objet (le savoir), mais il doit réinventer ce qu'il est seul à pouvoir faire: sa relation à l'objet. Tout ceci est une opération concrète, car elle s'effectue dans et par le langage qui sert à la fois de support et d'outil à ce travail d'appropriation » (Chabanne, Dunas, 1999 : 17).

Par conséquent, le postulat qui sous-tend notre démarche revient à dire que la pensée scientifique (abstraction, distanciation), l'imagination (pensée métaphorique, rêverie, fiction) et l'expérience (versant matériel et sensuel de l'activité d'écriture, expérience individuelle de la réalité) ne sont jamais séparées, mais fonctionnent au contraire en interaction permanente dans le processus d'élaboration des connaissances. Car à l'instar de Frédéric François, nous pensons qu'il n'y a pas d'opposition entre le senti, l'imaginaire et la « pensée réflexive » (2006: 221).

C'est ce que nous allons tenter de montrer ici en explorant cette « créativité ordinaire » (Chabanne, Dunas, 1999) qui nous parait constituer le cœur du mécanisme d'appropriation du savoir scientifique dans toute sa diversité.

\section{Le dispositif mis en place}

Notre dispositif s'inspire de celui proposé par Sauzeau et Triquet (2004). Ce dispositif, («l'atelier d'écriture d'un récit de fiction scientifique ») est présenté comme étant susceptible de contribuer à l'articulation entre « singulier » (pôle de la subjectivité) et typique ou générique (pôle de l'objectivité scientifique). Selon les auteurs «le traitement narratif d'un contenu scientifique serait propre à développer chez les scripteurs - simultanément et de façon interactive - des compétences identifiables sur le plan notionnel, en sciences, et sur le plan de la maîtrise du récit en français, en particulier au niveau de la compétence descriptive ". L'hypothèse est la suivante : "le fait d'avoir à transposer des notions scientifiques dans un nouveau genre permet au scripteur de les désolidariser de leur signifiant premier et ainsi de mieux les objectiver. C'est le pouvoir heuristique de cette procédure » (Triquet, Sauzeau, 2004 : 5). Dans le sillon de Bruner, les auteurs font le pari que le dispositif amène le scripteur à considérer la tâche comme une situation-problème : "la fiction amène à reconsidérer ce qui est évident, à bousculer nos habitudes à l'égard de ce que nous tenons pour la norme [...]. Une histoire commence lorsqu'apparaît une brèche dans l'ordre des choses : un grand récit [...] invite à poser des problèmes " (Triquet, Sauzeau, 2004 : 6).

Dans le cadre de notre propre dispositif l'objectif est d'aider nos étudiants à articuler les connaissances abordées en cours à leur propre histoire, à incarner le savoir dans une fiction pour lui donner forme et sens. 


\section{Contexte et objectifs du dispositif}

Le dispositif s'adresse à des étudiants inscrits en première année de Sciences du langage dans le cours « Pratext» dont l'objectif, comme son nom l'indique, est de «pratiquer» des textes de la spécialité, soit en situation de réception (lectureinterprétation), soit en situation de production, donc de travailler la compétence scripturale des étudiants en articulant maîtrise méthodologique et construction de connaissances disciplinaires. Toute l'année est construite autour de 4 thématiques (Origines des langues, Acquisition du langage, Naissance et mutations de l'écriture, Traitement automatique des langues). On travaille alternativement le pôle lecture et le pôle écriture. Les modalités pédagogiques du cours visent à articuler fortement savoirs (construction de connaissances, de repères dans un champ scientifique), savoir-faire (objectifs méthodologiques concernant la compétence scripturale) et savoir-être (démystification de la connaissance théorique, prise de confiance, appropriation des connaissances par le sujet, construction d'un point de vue).

La progression annuelle aboutit au semestre 1 à la constitution en binôme d'un dossier documentaire sur l'une des 4 thématiques. Le semestre 2 est quant à lui finalisé par la production d'une fiction scientifique, toujours sur l'une des quatre thématiques. Dans le cadre de cet article nous ne nous intéressons qu'aux productions réalisées au semestre 2 .

Au-delà de ces objectifs, le but est aussi de lutter contre l'insécurité scripturale massive en début de premier cycle universitaire, de faire émerger les « connaissances ignorées » (Penloup, 2007) de nos étudiants dans le domaine de l'écriture, de susciter leur créativité et de les engager dans et par l'écriture sur le chemin de la connaissance.

\section{Le récit de fiction : les caractéristiques du genre}

Le texte à produire comporte une fiction (histoire inventée), une narration (situations, personnages fictifs ou mise en scène des protagonistes de différents débats), et il doit par conséquent distraire le lecteur.

Mais c'est aussi un récit qui met en scène et incarne, en plus de la fiction, des idées, un point de vue sur une question, un problème scientifique, et il doit donc également permettre d'instruire le lecteur : l'information scientifique doit y être présente sous différentes formes et l'on doit par conséquent trouver dans cet écrit des extraits du discours scientifique mis en scène : «bribes» de textes (citations), notions, idées reformulées, arguments, courants théoriques, noms d'auteurs, dates, etc., qui vont être injectés au fil de l'histoire...

Ces deux impératifs (distraire et instruire) doivent trouver un équilibre dans le texte ${ }^{(2)}$.

Le corpus total recueilli sur les deux années est de 36 textes.

\section{L'atelier d'écriture comme tremplin}

Il nous a paru intéressant de préparer et d'accompagner ce travail d'écriture de fiction grâce à des séances d'atelier d'écriture. Quelles que soient les méthodes

(2) Voir à ce propos les critères de réussite proposés aux étudiants lors du deuxième atelier d'écriture, annexe 1. 
d'animation ${ }^{(3)}$, on sait que les ateliers facilitent le passage à l'écriture, aident les participants à reprendre confiance en leurs compétences scripturales et favorisent le travail de la réécriture (Lafont, 1999). Habituellement, dans un atelier d'écriture poétique, le jeu des activités et des consignes est destiné à contrarier l'activité consciente et réfléchie des scripteurs dans l'élaboration du texte. Le but de l'animateur est alors d'aider les participants à dépasser les représentations qu'ils ont des mécanismes de l'écriture ou de leurs propres compétences scripturales. Les associations libres, les échanges, le hasard, le travail sur le pôle matériel, l'ambiguïté volontaire des consignes doivent aider les participants à découvrir de nouvelles dimensions de l'écriture et à se découvrir des compétences nouvelles. Mais, le cadre de notre expérience impose un fonctionnement différent. La portée argumentative, démonstrative et scientifique du texte à produire exige des scripteurs un contrôle précis des contenus et de la signification du texte final. Les séances d'atelier doivent donc travailler dans deux directions. Tout d'abord, il faut « libérer » l'écriture des participants en montrant des mécanismes de l'écriture littéraire et en autorisant les fantaisies de l'imagination. C'est là le rôle traditionnel des ateliers d'écriture. Mais ce travail doit être mené en parallèle à la construction d'un contenu scientifique. C'est pourquoi les trois séances d'atelier ont associé ces deux dimensions.

Lors de la première séance, les participants ont été invités à choisir parmi les quatre thèmes de l'année celui qu'ils souhaitent développer dans leur fiction scientifique. Chacun choisit ensuite le mot qui lui semble essentiel et qui est ensuite développé sur les deux axes du signifiant et du signifié. Les listes obtenues sont ensuite tissées pour créer des expressions. Voici à titre d'exemple, la liste des expressions de Chloé qui travaillait le mot «mémoire » : "le déboire du souvenir, l'intelligence de l'histoire, le souvenir noir, séchoir de l'intelligence, mémori du disque dur, le trou du loir, la mouvance de la retenue, le mémo d'Alzheimer. " Certaines des ces expressions sont ensuite mises en commun et analysées pour montrer comment ce procédé d'écriture permet de construire du sens sur l'objet travaillé. Un certain nombre d'expressions qu'on retrouve dans les textes finaux sont issues de cette phase du travail. A la fin de la séance, les participants produisent, à partir de ce matériau, une définition du thème qu'ils ont choisi. Les textes présentent toutes les caractéristiques des textes produits en atelier : associations lexicales inattendues, jeu sur les sonorités, utilisation de la métaphore. Tout 1'intérêt consiste à faire prendre conscience aux participants que ces textes "poétiques », si différents de ceux produits habituellement à l'université, sont porteurs d'une idée sur le thème travaillé.

Nous avons construit le second atelier en deux temps. D'abord, un travail d'exploration du contenu scientifique des dossiers documentaires pour faire extraire aux étudiants, sous forme de tableau, les informations scientifiques de chaque dossier (voir annexe 2). Ce tableau constitue, au moment de l'écriture, une banque de données à intégrer dans la fiction, qu'il s'agisse de personnages, d'éléments historiques ou théoriques. Ensuite, nous avons travaillé sur la construction du personnage principal. L'atelier s'achevant sur le portrait de ces « héros ». Les exemples de portraits montrent qu'à ce stade du travail, les principaux éléments du texte final sont déjà en place.

(3) Nous utilisons la méthode issue des travaux du GFEN. Voir par exemple NEUMAYER, M., NeUmayer, O., Animer un atelier d'écriture, Faire de l'écriture un bien partagé, ESF, Paris, 2003. 
La troisième séance a été consacrée à l'élaboration du synopsis de nouvelles. L'atelier a commencé par un travail de formulation des problématiques qui seront illustrées par les récits. Ensuite, les participants ont élaboré le synopsis de leur fiction en s'appuyant sur différents modèles de structuration du récit (Schéma quinaire; schéma actantiel). Nous n'avons pas imposé ces modèles aux étudiants, mais nous les avons présentés comme des aides facultatives pour la création de leurs scénarios. Ceux-ci ont ensuite été affinés grâce à un travail de lecture collective et de réécriture.

En définitive on peut dire que la démarche mise en place a pour but de travailler en parallèle les deux pôles de l'écriture : le pôle rationnel de la « raison graphique » visant la construction de connaissances objectives et faisant référence au « mode paradigmatique » décrit par Bruner, et le pôle subjectif de la " création graphique », correspondant lui au « mode narratif ». Au moment d'évaluer ces productions, nous avons exploré les textes à la recherche des graines semées durant l'atelier, en espérant y voir germer quelques fleurs de science.

\section{Analyse des résultats}

Les étudiants se sont prêtés de bonne grâce à ce dispositif d'un genre nouveau, peu conforme en tout cas aux routines universitaires et aux habituels « devoirs » de fin d'année dans lesquels on leur demande en général de restituer de façon monographique le savoir dispensé en cours durant l'année. Ici la consigne portait sur la capacité à se saisir des connaissances travaillées ensemble pour créer un texte autonome, et plus particulièrement une fiction.

Qu'apporte ce travail ? En quoi l'atelier d'écriture et le travail sur la fiction a-til participé concrètement à la construction d'un savoir scientifique ? Sous quelles formes se présente ce savoir? Quelles conceptions du discours scientifique ce travail a-t-il permis ? Quelles difficultés y sont rencontrées ? Quelles visées énonciatives y sont développées ? Quels types de compétences scripturales sont à l'œuvre ? Quelles qualités littéraires s'y révèlent?

Nous ne répondrons pas ici à toutes ces questions. L'analyse des productions va se centrer sur deux points saillants : la construction du point de vue au fil du récit, et l'intrication profonde dans les textes entre mode paradigmatique et mode narratif.

Les premiers éléments d'analyse du corpus permettent de faire émerger les formes hétérogènes et inattendues que peut prendre la connaissance scientifique, la transformation progressive des conceptions du discours scientifique. En ouvrant les vannes de l'imagination par la fiction, les récits amènent les étudiants à dépasser les limites du « savoir objet » et d'aller au-delà de l'horizon d'attente initialement visé...

\section{La construction d'un point de vue sur le terreau des représentations}

On constate fréquemment chez les étudiants de première année une difficulté à définir, construire et assumer un point de vue sur les " connaissances théoriques ». Ils se plaignent, paradoxalement, qu'elles sont abstraites. Or, la construction de représentations personnelles des objets étudiés est une clé importante de progrès et de réussite dans les études puisqu'elle constitue le préambule à toute appropriation des savoirs. On peut donc voir quel rôle l'écriture d'une fiction joue dans l'émergence des représentations et dans la construction d'un point de vue personnel sur le contenu scientifique des dossiers documentaires. 
Nous reprendrons, pour identifier et analyser la genèse de ce point de point de vue, les trois pistes de travail proposées par F. Audiger et C. Ronveaux (2007). La première piste est centrée sur le narrateur, et s'intéresse, dans la perspective de notre travail, à « la question du point de vue et des ressources sur lesquelles s'appuie le narrateur pour décrire une action, raconter un enchaînement d'actions [...] ». La seconde piste concerne « la construction des imaginaires sociaux », c'est-à-dire la façon dont le scripteur d'un récit de fiction utilise les sources historiques ou scientifiques pour rendre son récit « vraisemblable ». La troisième piste s'intéresse quant à elle à la « reconstitution du référent », c'est-à-dire « la réalité, les expériences du passé, les événements, etc. »

Ces trois axes nous semblent de bons outils pour faire émerger et observer les représentations à l'œuvre dans les récits et évaluer la construction d'un point de vue chez les étudiants. En effet, le monde de référence des fictions scientifiques sera constitué, en grande partie, d'éléments des dossiers documentaires, et donc de savoirs historiques et scientifiques. Pour produire un récit cohérent, vraisemblable, les étudiants devront donc élaborer un point de vue personnel à partir de ces contenus et en fonction de leurs représentations de ces contenus.

\section{Margaux : une construction efficace des objets de savoir dans la narration.}

Le récit inventé par cette étudiante nous fait suivre, à la première personne, les étapes de l'acquisition du langage de Sally, de la naissance à un an. Ce thème est associé à un point de vue (le langage s'acquiert dans les échanges sociaux, par imitation) ainsi qu'à un élément de fiction (Sally est guidée dans ses apprentissages par une nourrice de conte de fées). Une combinaison des trois axes énoncés plus haut permet à l'étudiante de construire dans son récit une double cohérence, narrative et cognitive.

Pour ce qui est des ressources mises en œuvre dans la narration, les connaissances scientifiques du dossier structurent chronologiquement le récit. Cela commence avec les échanges intra-utérins : "jusque là tout était noir et je n'entendais que les sons rassurants de la voix de ma mère et du battement de son cœur. " Au bout d'environ un mois, 1'enfant prononce ses premiers sons : «Je ne le contrôlais pas le moins du monde mais j'entendais ce bruit sortir de ma bouche grande ouverte: “haaaaa!" [...] c'était le premier véritable son quej'avais produit depuis ma naissance. " Ensuite, aux alentours de cinq mois, l'enfant accède au prélangage : "grâce à l'injection du pédiatre, tu pourras communiquer avec moi grâce à des regards et comprendre ce que les sons signifient. [...] ... Un son étrange sort de ma bouche et je la regarde intensément. Nourrice se met à sourire et me regarde tendrement. "Oui ma petite Sally, nous allons monter dans la voiture." C'est la première fois que j'arrive à me faire comprendre. " La progression se poursuit jusqu'au premier mot, maman, bien sûr, aux alentours de onze mois. La place manque ici pour suivre avec précision dans le texte de cette étudiante le récit des différentes étapes de l'acquisition du langage. Elle travaille à partir des documents du dossier documentaire, en opérant une synthèse et une sélection cohérente des informations sur ces étapes. Lorsqu'on observe la structuration temporelle on constate que le récit les respecte, la partie scientifique est donc pertinente. Par contre, il y a une contradiction dans la structuration temporelle de la fiction puisque la petite Sally naît le « 16 mai 2009 à $11 \mathrm{hl} 8$ » et qu'un peu plus loin on trouve : "l'été arrive [...] j'ai bientôt sept mois». La cohérence scientifique serait-elle plus simple à respecter que la cohérence narrative? 
Par ailleurs, grâce à la fiction l'étudiante reconstruit un monde de référence. Elle puise dans l'imaginaire social une situation connue et acceptable : des parents accaparés par leur travail qui confient leur bébé à une nounou, ce qui autorise une approche psychologique autour des thèmes de la séparation et de la culpabilité : "Le soir, quand maman est venue me chercher, j'étais tout excitée à l'idée de lui montrer ce que j'avais appris à faire. [...] Elle s'est mise au volant et m'a demandé comment s'était passée ma journée. Un large sourire s'est installé sur mon visage, j'allais pouvoir lui montrer! Ma bouche commençait à s'ouvrir quand tout à coup, le portable de maman a sonné... ». La fiction trouve ainsi un cadre vraisemblable.

Parallèlement à ces informations sur les étapes de l'acquisition du langage, le récit permet de développer un point de vue plus personnel sur les conditions optimales de cette acquisition. Comme angle d'attaque, l'étudiante a donc choisi de se focaliser sur le rôle de l'imitation et des échanges sociaux. A chaque nouvelle étape, le récit met en scène l'interaction et l'imitation qui permettent à l'enfant d'entrer progressivement dans le langage : "J'ai observé ses lèvres quand elle a parlé, sans le vouloir et elles ont bougé dans tous les sens, c'est bizarre! [...] c'est décidé, je vais essayer de bouger mes lèvres comme elle. " La position de l'étudiante est résumée à la fin de la nouvelle par la nourrice : "laissez-la vous imiter tout le temps [...] Plus vous lui parlerez, plus elle apprendra vite. Quant à la structure des phrases, chaque enfant a, en lui, dans une petite partie de son cerveau, le schéma d'une grammaire universelle qui l'aide à devenir un vrai petit roi du langage. "Ce point de vue sur les conditions d'une bonne acquisition du langage peut constituer l'amorce d'un positionnement professionnel (beaucoup des étudiants de Sciences du Langage veulent devenir orthophoniste), aussi bien qu'une posture pour aborder les connaissances théoriques ultérieures.

Ainsi, dans le cas du récit de cette étudiante, c'est bien la mise en relation cohérente des connaissances et des représentations qui permet de déboucher sur l'affirmation d'un point de vue personnel. Ce qui est déjà un pas important, même s'il n'est pas sûr que l'étudiante puisse le formuler clairement. C'est un point qui reste à vérifier. Le récit permet donc d'organiser ce bric-à-brac d'images et d'objets de savoir parce que le scripteur est libre de déterminer son parcours et c'est cette liberté qui permet de construire un chemin personnel pour tirer du bric-à-brac un nouvel objet, la fiction scientifique, qui pourra être utilisé/lu s'il est réussi. Ce qui n'est pas toujours le cas.

\section{Raphaëlle : un positionnement problématique qui débouche sur une impasse narrative.}

Cette étudiante a choisi de traiter le thème de l'invention de l'écriture. Son récit pose un double problème, de construction et de développement, parce qu'il s'appuie sur une lecture et une compréhension fragmentaires et mal orientées du dossier documentaire.

Le premier problème relève du dosage des éléments de narration-fiction et de ceux qui concernent la problématique scientifique. Son texte est structuré en trois parties à peu près égales : une présentation du héros, Eroïmé, sa rencontre magique avec le dieu égyptien Teuth, inventeur de 1 'écriture, et d'un dialogue entre les deux personnages. Au cours de ce dialogue, Teuth dénonce les conséquences néfastes de l'invention de l'écriture sur la mémoire humaine. La présentation du personnage, Eroïmé, occupe un tiers de l'ensemble et plusieurs indices montrent 
que ce long portrait est issu presque directement du travail d'atelier. Le texte commence par la phrase de Vian qui avait servi d'inducteur au moment de l'écriture du portrait du personnage "Erö̈mé terminait sa toilette»; ses caractéristiques - passion pour le jardinage, rupture familiale, solitude amoureuse, rêves prémonitoires - sont ensuite énumérées sans être mises en relation avec la problématique scientifique qui sera traitée. A aucun moment de ce portrait n'apparaît la question de l'invention de l'écriture.

Il faut attendre le dernier tiers du récit pour trouver des références explicites aux éléments du dossier ainsi qu'à la problématique choisie. Le récit se présente donc comme la juxtaposition de deux textes. La partie centrale qui raconte la rencontre des deux personnages et la justifie dans l'ordre de la narration est une simple transition. Le portrait initial, construit au début du travail d'atelier, n'a pas été revisité à la lumière de la problématique scientifique qui a été définie plus tard.

Dans cette dernière partie, par contre, les références aux éléments du dossier sont nombreuses ( 6 en tout), mais ces éléments sont travaillés de telle manière qu'ils conduiront à une impasse scientifique et narrative.

La première question théorique abordée par l'étudiante est celle des origines de l'écriture. Dans un premier temps, Eroïmé l'attribue aux Phéniciens : " c'est au Liban qu'elle est apparue et dans des besoins que le commerce a peu à peu installés. Les Phéniciens ont eu besoin de l'écriture pour noter les différentes marchandises, établir des listes et ont inventé des contrats commerciaux. A partir de là, la parole des gens ne suffisait plus ». L'étudiante reprend ici des éléments des documents 1 et $2^{(4)}$ qui sont ensuite mis en opposition avec le texte de Platon (document 6 du dossier) et considérés comme faux puisque Teuth répond: "Tu commets là, Erö̈mé, une grande erreur. Je ne peux point t'en vouloir car la majorité des gens pense de la même manière que toi. Mais cela reste une bien mauvaise réponse. " Le dieu égyptien reprend ensuite à son compte les affirmations de Socrate $^{(5)}$ sur l'invention de l'écriture : "Parce que c'est moi qui l'ai inventé en Egypte il y a fort longtemps. Tu n'as donc pas étudié les grands philosophes tels Socrate?»

On constate ici de la part de l'étudiante une double confusion. Sur le plan épistémologique, tout d'abord. Le récit ne distingue pas l'invention des hiéroglyphes et celle de l'écriture alphabétique. Dans le dossier, les deux étapes de l'invention de l'écriture ne s'opposent pas mais se succèdent et se complètent. L'étudiante semble n'avoir pas conscience de la spécificité des apports de l'écriture alphabétique. La seconde confusion porte sur la nature des documents puisque le texte de Socrate est mis sur le même plan qu'un document scientifique et historique récent. Il n'y a donc pas de regard critique sur la validité scientifique des informations apportées par les documents du dossier.

Après la question des origines de l'écriture, le récit aborde un second thème,

(4) «Il a été mis au point par les Phéniciens, un peuple de commerçants navigateurs habitant sur les côtes de l'actuel Liban» (Chignier, J. et al., 1990, pp. 1-15).

«Les débuts de l'écriture répondent donc à des fonctions comptables et commerciales. A cela vont s'ajouter des préoccupations administratives et politiques... », (Weinberg, 2000, pp. 22-25).

(5) «J'ai donc ouï dire qu'il existait près de Naucratis, en Égypte, un des antiques dieux de ce pays, et qu'à ce dieu les Égyptiens consacrèrent l'oiseau qu'ils appelaient ibis. Ce dieu se nommait Theuth. C'est lui qui le premier inventa la science des nombres, le calcul, la géométrie, l'astronomie, le trictrac, les dés, et enfin l'écriture ", (Platon, Phèdre, http ://fr.wikisource.org/wiki/phèdre). 
celui des conséquences de cette invention. Cette fois, le récit de l'étudiante rapproche les arguments du texte de Platon d'informations extraites de l'article d'Achille Weinberg (2000), qui présente les théories de J. Goody. L'Egyptien Theut explique qu'il a inventé l'écriture « dans le but de soulager la science et la mémoire [...] et que cela permettrait d'avoir un espace illimité pour stocker toutes nos informations. " Ces passages sont des citations à peine transformées de l'article de A. Weinberg ou du texte de Platon. Ce rôle de " mémoire externe » de l'écriture est alors opposé aux arguments de Socrate contre l'écriture. D'après Socrate, le roi Thamous aurait dévoilé à Teuth les dangers de l'écriture. Ces arguments sont repris dans le récit de l'étudiante : "...l'écriture serait un danger. Un danger car nos âmes oublieront ce qu'elles savent et nos mémoires seront de plus en plus négligées par le fait que nous pourrions nous reposer sur l'écrit. » Le débat débouche sur un constat alarmiste. Depuis l'invention de l'écriture, on assisterait à " une énorme perte des capacités de mémoire humaine. "

L'utilisation de l'expression "capacités de mémoire humaine » indique que l'étudiante s'est limitée à une représentation physique de la mémoire, la quantité de souvenirs et de connaissances qu'elle peut contenir. Cette vision est réductrice parce que, dans le texte de Socrate, si cette notion d'oubli, de «perte de capacité » est évoquée, elle est aussitôt complétée : «Parce qu'ils auront foi dans l'écriture, c'est par le dehors, par des empreintes étrangères, et non plus du dedans, du fond d'eux-mêmes, que les hommes chercheront à se ressouvenir " ${ }^{(6)}$. La critique socratique des conséquences de l'écriture sur la mémoire ne porte donc pas seulement sur sa capacité, mais surtout sur le rôle qui lui est accordé, avec l'opposition entre le « dehors » et le « dedans ». La suite du dialogue développe d'autres critiques sur l'écriture qui ne sont pas non plus prises en compte dans le récit. Il y a sans doute chez cette étudiante une représentation initiale de la mémoire, perçue comme un contenant et non comme un outil, qui empêche de voir la véritable nature des arguments de Socrate. D'autre part, on peut se demander si le constat négatif d'une humanité en décadence qui s'exprime dans le récit ne trouve pas son application/implication dans le discours ambiant sur la baisse du niveau ? Cette représentation empêchant d'entrer dans la complexité du savoir.

Le second problème posé par le récit de cette étudiante découle de ce constat qui est, de fait, un cul-de-sac narratif puisque le récit s'arrête quelques lignes plus tard. La dernière réplique d'Eroïmé exprime clairement ce désarroi de la fiction : "Je crois comprendre ce que tu veux dire et ce que cela représente mais que puisje y faire? " La réponse de Teuth, qui clôt le récit, laisse entendre qu' une suite est possible: "Suis-moi, je vais t'expliquer en quoi va consister ton rôle mais il faut que tu sois prêt pour un long et fatigant travail. Nous ne pourrons pas faire comprendre tout cela du jour au lendemain. " Mais cette suite n'est pas développée alors qu'elle aurait pu/dû prolonger la réflexion sur la place de l'écriture dans notre société. On peut supposer que cette impasse narrative repose sur une compréhension parcellaire des enjeux du dossier, et particulièrement du texte de Platon qui fournit au récit l'essentiel des arguments et un protagoniste.

La comparaison de ces deux textes, de leurs réussites et de leurs échecs, montre que le passage par le récit n'est pas une martingale qui permettrait de corriger automatiquement les représentations erronées, les approximations scientifiques ou les problèmes méthodologiques des étudiants. Par contre, il nous semble que le récit a un rôle spécifique à jouer en parallèle avec les autres écrits universitaires.

(6) Platon, op. cit. 
La fonction implicative du récit, pour reprendre les termes d'Yves Reuter, qui permet et favorise l'implication du sujet scripteur dans son texte, en fait un outil efficace pour faire émerger les représentations qui peuvent alors être formalisées, conscientisées et discutées dans l'optique d'une évolution.

\section{Les récits des origines ou l'intrication entre mode paradigmatique et mode narratif}

"Il s'agit en effet de trouver l'action des valeurs inconscientes à la base même de la connaissance empirique et scientifique. Il nous faut donc montrer à la lumière réciproque qui va sans cesse des connaissances objectives et sociales aux connaissances subjectives et personnelles, et vice versa. Il faut montrer dans l'expérience scientifique les traces de l'expérience enfantine ».

(Gaston Bachelard, La psychanalyse du feu, 1949, réédition 1986 : 23-44).

Dans une étude menée il y a quelques années (Frier, 2004), nous avions montré les difficultés récurrentes de nos étudiants à articuler dans leurs rapports de stage savoir théorique et expérience de terrain. Nous relevions notamment une tendance à rapporter des faits non problématisés, à «plaquer » un fondement théorique mobilisé de façon très ponctuelle, un manque d'implication, un effacement du scripteur peu propice à l'argumentation et au développement d'un point de vue personnel.

Dans le corpus recueilli cette année quelque chose a changé fondamentalement et il semblerait que la fiction empêche la production de telles « monographies immobiles »c'est-à-dire de textes se caractérisant par un discours de description, de définition, sans véritable questionnement heuristique. Les récits que nous appelons ici « récits des origines » sont de loin les plus nombreux de notre corpus, quele que soit la thématique choisie. Cela nous a frappés. Ils réunissent, à des degrés divers les trois visées énonciatives décrites par Bautier et al. (2000) : monographique (description, définition, tabularisation), explicative (chronologie et causalité), argumentative (présence d'un point de vue). En effet tous ces textes font cohabiter, chacun à sa manière, les éléments "objectifs » de la connaissance scientifique (définitions, noms d'auteurs, de théories, de courants, dates, etc.), les éléments de la fiction qui sont censés apporter un cadre et une explication à la question posée par le texte, et les éléments de l'argumentation (point de vue défendu). La présence systématique de ces trois visées est très significative à nos yeux car à l'instar de Bautier et al., nous sommes convaincus que "les élèves qui réussissent à l'école sont ceux qui sont capables de circuler entre plusieurs visées, plusieurs postures langagières » (Bautier et al., 2000 : 160). L'exploration de ces récits nous amène à penser que cette mobilité dans le domaine des apprentissages scientifiques est fortement favorisée par le recours à la fiction. Voici en guise de preuve quelques joyaux de l'atelier.

\section{Julie : aux origines de l'écriture}

Cette fiction incroyable nous plonge de façon très réaliste dans l'actualité universitaire du mois de mai 2009, vers la fin du mouvement de grève. Le thème du récit est la naissance de l'écriture. La thèse défendue concerne le rôle structurant de l'écriture sur la pensée et se réfère aux théories développées par Jack Goody. L'héroïne de l'histoire est une étudiante, emprisonnée suite à une rafle, qui du fond de sa cellule, cherche à comprendre ce qui lui arrive, qui a trahi et pourquoi. Mais en vain, car privée de tout, et surtout d'écrits, elle ne parvient pas à mettre de 
1'ordre dans ses idées : « sans support, je n'arrivais pas à trier mes pensées. Ma colère troublait mes idées, la fatigue aussi. J'avais besoin de poser les choses, de classer mes souvenirs, de structurer ma mémoire ». La quête de l'héroïne est donc de pouvoir écrire, car seule l'écriture pourra l'aider à retrouver son passé, à penser, à avancer. La situation-problème posée par ce récit est donc clairement identifiée dès le départ et dans une double perspective : comment trouver un moyen pour écrire ? Comment retrouver la source primitive de l'écriture?

La visée explicative du texte fait alterner deux types de réponses : un premier ancré dans la matière et la rêverie : "Depuis quelques jours, machinalement, j'avais gratté avec ma cuillère, dans le bois mou de la vieille planche qui me servait de couche des petits batons afin de compter les jours et tenter de retrouver des repère dans le temps. J'avais fait cela sans réfléchir. Ce grattage graphique primaire était l'unique façon de m'occuper les mains et l'esprit... Je m'imaginais dans une grotte préhistorique, grattouillant des petits mammouths et esquissant les premiers pas de l'écriture...".

On peut souligner à quel point cet extrait rappelle d'un point de vue psychanalytique le retour aux limbes, le retrait dans la matrice originelle qui précède la pensée rationnelle.

Le second type de réponse renvoie à une connaissance objective : "Je me rappelai soudain d'un texte de Jack Goody, un anthropologue anglais qui, à la suite de son emprisonnement en Italie, durant la seconde guerre mondiale, avait écrit un livre sur l'écriture. Il me semblait bien me souvenir qu'il y évoquait la difficulté de penser et de rassembler ses idées sans le support de l'écrit. Il avait expliqué dans "the consequence of literacy" la véritable mutilation intellectuelle qui représentait l'impossibilité de lire ou d'écrire... Je tenais le remède à ma torture d'érudite!».

D'où l'idée de se procurer de la terre, en cachette, à l'occasion de la sortie hebdomadaire dans la cour de la prison pour fabriquer avec cette pâte des plaques de glaise pour écrire : "Je me jetai à plat ventre sur le sol et commençai à gratter la terre humide pour l'enfourner par grosses poignées dans les poches de mon pantalon et de ma veste. [...]. Du bout de ma cuillère pliée, j'enfonçais mon alphabet dans la matière [...]. Je me sentais moderne sumérienne en Mésopotamie, gravant mes tablettes d'argile, 3500 avec Jésus-Christ!".

Là encore dans cet extrait on voit très nettement ce va-et-vient régulier dans le texte entre une pensée métaphorique, onirique, ancrée dans la matérialité des éléments (ici la terre), et une pensée plus rationnelle, fonctionnant à partir de repères stables (dates, nom d'auteur, titre d'ouvrage, concepts) et de faits objectifs.

La chute du récit excelle en la matière et réussit à mélanger de façon presque inextricable arguments objectifs, empreinte de l'expérience concrète, et traces de la rêverie initiale : "Je recommençais ainsi mes mixtures cunéiformes avec chacun des tas que j'avais conservés sous mon lit, puis quand les tablettes étaient remplies et bien sèches, je les stockais sous ma couverture, à l'abri. Chaque graphème ancré dans la terre me libérait encore un peu l'esprit, et laissait la place à une réflexion plus large. Je pouvais lister des noms, reformuler mes notes, me souvenir des détails sans encombrer davantage ma mémoire... Tout devenait enfin plus visuel et plus clair. Je pouvais aisément comprendre comment notre "civilisation de l'écrit" avait pu être un bond intellectuel pour l'homme, je vivais cette révolution en direct! Les longs trains darwiniens de mes pensées se démontaient et se remontaient à ma simple demande. C'était comme si je mettais mon cerveau 
dans un ordinateur et que j'imprimais tout ce qu'il y avait dedans, ainsi, je pouvais effacer de ma mémoire ce qui était devant mes yeux, et cela laissait le double de la place originelle! J'avais compris la technologie des mots ... mais surtout, le nom du traître me sautait désormais à la figure. "

\section{Sandy : l'aventure du premier mot de Médes ou quand Chomsky devient un poisson rouge}

C'est un magnifique récit, plein de trouvailles et d'humour, et qui fait la part belle à quelques «pépites » de l'atelier, réinvesties au fil du texte. Le thème choisi est celui de l'acquisition du langage. C'est l'aventure du premier mot prononcé par Médes, un petit garçon d'un an à peine qui veut aider sa maman à retrouver ses clés jetées dans l'aquarium des poissons par sa grande sœur Linda. La problématique défendue fait alterner thèse innéiste et interactionniste, en mettant l'accent sur l'élan (presque physique) qui crée le désir d'apprendre à parler chez l'enfant. Toutes les étapes de l'acquisition du langage sont reprises et intégrées de façon très judicieuse à partir du point de vue de l'enfant, dont les efforts pour se faire comprendre donnent au récit un caractère réaliste très frappant : " $j$ 'avais déjà franchi la première étape : créer une situation propice à la communication en captant l'attention de mon interlocuteur. C'était d'ailleurs une situation assez agréable que de sentir cette proximité avec ma mère. Je venais de comprendre qu'on peut fonctionner à deux, et cela m'intriguait. A travers ce babil encore "sauvage" que je maîtrisais depuis quelques semaines, j'étais entré dans ce que l'on appelle le "prélangage”. Mais malheureusement cela n'était pas suffisant. Bref, ma réponse se limita à un léger mouvement de la lèvre accompagné par son délicat coulis de salive!"

Tentative vaine donc puisque Médes n'atteint pas son but : "Quelques bababa et dididi après, oui, parce qu'à force d'entraînement, j'étais maintenant capable de répéter des syllabes, un vrai pro du babillage canonique, à seulement six mois! (Pas mal non?), maman m'annonça qu'il était l'heure de la sieste ».

Médes se retrouve donc au lit, et c'est dans la pénombre de sa chambre, propice à la rêverie, qu'il va trouver la solution à son problème : "Je me retrouvais là, dans cette pièce aux couleurs assombries par les rideaux opaques que maman venait de fermer. Seuls quelques rayons de soleil les traversaient encore, laissant apparaître des contrastes et des jeux de lumières apaisants dans toute la chambre. Je pouvais encore apercevoir les avions suspendus au plafond, juste au-dessus de mon berceau. Je sentais mes paupières s'alourdir de plus en plus, malgré mes efforts pour les maintenir ouvertes. J'eus un instant la sensation que les avions s'étaient mis à bouger, à valser au-dessus de moi. Un silence presque religieux régnait, et je me laissais emporter par cette atmosphère relaxante. Je finis par m'endormir. Alors plongé dans les bras de Morphée, je fis la rencontre de Chomsky, mon poisson rouge. J'étais moi aussi un poisson, et nous nous laissions aller tous les deux au rythme des vagues de l'océan. "Ne prends pas le large mon ami, car tu as encore beaucoup à faire. Lorsque tu te réveilleras, tu devras grandir en fanfare et accomplir ton devoir" "».

A son réveil, Médes est à nouveau envahi physiquement par sa volonté de communiquer avec sa mère et de lui faire comprendre où sont passées ses clés. C'est l'occasion de faire interférer dans le récit le caractère proprement sensuel et physiologique du désir de parler chez l'enfant et les connaissances objectives sur l'acquisition du langage : "Je fus sorti de mes songes par ce grand frisson qui par- 
courait encore une fois mon corps. Je sentais que tout se chamboulait en moi, comme si mes connaissances se décuplaient jusqu'à s'accumuler dangereusement... C'était une sensation étrange et inquiétante. Cette ébullition sourde était en train de me changer irrémédiablement et je me laissais faire, porté par ce vigoureux désir de rendre ses clés à maman. J'avais toujours eu cette sensation d'avoir comme un trésor enfoui en moi, une boite de pandore qui ne demandait qu'à être ouverte. Le trésor en question, j'en étais maintenant persuadé, c'était ce que Chomsky (le linguiste) appelle la grammaire universelle. J'avais en moi la boite, il ne manquait que la clé et la personne pour l'ouvrir. C'est ce qui m'arrivait en ce moment. Avec l'aide de Chomsky et d'une situation pour le moins particulière, j'avais provoqué le sort. Tout ce qui sommeillait en moi était en train de se réveiller et j'apprenais la langue familière qui me berçait depuis la naissance [...] Le quiproquo développemental que je subissais depuis le début de la journée venait de prendre tout son sens $"$.

A la fin du texte, lorsque Médes prononce enfin son " premier mot», les éléments de connaissance objective (aspects interactionnels du langage, gestion de l'attention conjointe, fonction du geste de pointage, étayage de l'adulte, zone proximale de développement) s'articulent de façon très habile aux conditions proxémiques de l'échange (jeux des regards, mouvements des corps, enjeux affectifs de la situation de communication) : "De la main gauche, je pointais l'aquarium. Les yeux plongés dans ceux de maman, je dirigeais son regard jusqu'à fishland. Jusque là, tout fonctionnait à merveille! Maman s'approcha alors avec délicatesse. Je secouais plus rapidement la main gauche portant les clés, tout en pointant de la droite le fond de l'aquarium, l'endroit exact où se trouvaient ses clés. Je prononçais à cet instant mon premier mot : "asson" (à traduire poisson, mais avec l'excitation... !). Pourquoi "poisson"? Et bien parce que sans mon poisson rouge je ne serais arrivé à rien. Parce que sans Chomsky, je n'aurais jamais découvert les joies du langage - bien que je n'aie pas encore tout exploré. Cependant, j'avais eu un aperçu de ce que l'on ressent lorsqu'on communique avec les autres et du bonheur que cela peut procurer. On dit que le premier mot d'un bébé évoque toujours quelque chose de concret, quelque chose qui lui soitfamilier. Ce fut mon cas. Lorsque je tournais à nouveau le regard vers Chomsky pour le remercier, celui-ci, bouche bée, libérait bêtement quelques bulles qui remontaient jusqu'à la surface pour exploser en silence. Maman venait de comprendre. Elle sourit et sur un ton enjoué elle lança: "Mes clés! Mes clés étaient dans l'aquarium!"”.

A nouveau, ce qui fait l'intérêt à la fois littéraire et scientifique de ce texte, c'est cette capacité à incarner des éléments de connaissance objective dans le langage, à mêler par la fiction expérience concrète, réminiscence émotionnelle et onirique de la petite enfance et savoirs théoriques, donc à faire fonctionner de concert mode paradigmatique et mode narratif.

\section{Marie : l'amour à l'origine des langues du monde}

Ce récit traite de la question de l'origine des langues du monde. La problématique est posée d'emblée au tout début du texte : "T'es-tu déjà demandé d'où viennent les langues du monde? Il y a environ 6800 langues parlées sur cette planète, impressionnant n'est-ce pas? Mais d'où viennent-elles? Qui les a créées, comment, quand et dans quel but? ". Le débat opposant monogénèse et polygénèse est également présenté dès les premières lignes du texte, ainsi que le point de vue de 
l'auteur : "Pour moi la thèse de la monogénèse (c'est-à-dire qu'une unique langue aurait engendré toutes les autres) est la plus valable. Après tout, si on postule la thèse de la polygénèse (dans ce cas plusieurs langues seraient à l'origine des toutes les autres), comment expliquer certaines ressemblances troublantes entre des langues parlées dans des pays pourtant géographiquement et culturellement éloignés?

L'enjeu du texte est alors de donner la « vraie réponse », c'est-à-dire la réponse scientifique à cette question : "Dans la Bible, l'histoire de Babel donne une explication du passage d'une protolangue unique à la multitude actuelle en évoquant un châtiment divin.

Nous ne sommes plus en 1860 et la Société de Linguistique de Paris n'interdit plus aucune recherche ou publication au sujet de l'origine des langues (il faut dire qu'à l'époque des thèses bien moins crédibles que Babel avaient été évoquées!).

Je voudrais donc te donner la version non religieuse des faits, la vraie version bien sûr alors, cher lecteur, voici pour toi l'histoire de la naissance de la Langue Mère!"

La réponse à cette question va être livrée par le biais d'un récit extraordinaire, très bien documenté, émaillé de détails géographiques, anthropologiques, paléontologiques très précis, qui emporte le lecteur en Afrique à une époque très lointaine : "Nous sommes entre 50.000 et 60.000 années avant notre ère. En Afrique dans un territoire qui correspond à l'actuel Cameroun vit un peuple que l'on appelle les Bantous. (Ce nom ne te ditrien? Je n'invente pas, les dates et le nom de ce peuple sont véridiques. Si d'ailleurs après mon récit tu t'y intéresses, je te recommande de lire quelques écrits de Greenberg). Ce matin-là, tandis que le soleil se lève à peine, les hommes vont sortir les chèvres de leurs abris alors un peu rudimentaires. En effet, depuis peu, les Bantous ont réussi à domestiquer des chèvres. Ils ont aussi mis en place les débuts de l'agriculture. Plus tard, ce peuple ainsi que d'autres en même temps que lui découvriront comment domestiquer les moutons, puis du bien plus gros bétail encore. Ce peuple Bantou découvrira aussi les techniques métallurgiques et pourra encore améliorer ses techniques agricoles.

Mais ce qui nous intéresse pour le moment est la langue que parlent cet homme qui attache une chèvre à un piquet, cet autre qui se lave dans la rivière ou cette femme qui montre à sa petite fille comment nouer ses cheveux. Oui, j'ai bien dit la langue et non le langage, car il me paraît important de bien discerner les deux concepts».

C'est l'histoire de Nigiro, un jeune homme qui va permettre à son peuple de franchir une étape fondamentale dans son évolution en inventant le premier mot d'une langue structurée. En effet jusqu'alors ce peuple communique de façon rudimentaire, en utilisant un protolangage fait de grognements et de mimiques. Un terrible incendie va contraindre le peuple de Nigiro à s'exiler et à partir à la recherche de nouveaux territoires. C'est à cette occasion, lors de cette quête, que Nigiro va rencontrer la "rousse demoiselle». Et c'est lors de cette rencontre qu'il invente le premier véritable vocable de l'humanité : "Son cour se met à battre si fort qu'il en est presque effrayé. C'est alors que, pour la première fois de l'humanité, Nigiro prononce... un mot. Ce n'est ni un grognement, ni un hoquet, ni un son venu d'on ne sait où mais bel et bien un mot. Ce mot, Nigiro vient de l'inventer, comme il en inventera mille et un autres par la suite, en écoutant son cour. Ce mot lui est apparu comme une évidence en voyant la rousse demoiselle. Tu sauras 
donc, cher lecteur, que le premier mot de l'humanité fut ce que l'on traduirait aujourd'hui par "belle" ".

Ce qui donne toute sa force à ce récit, c'est le fait d'incorporer les éléments de connaissance objective en leur donnant une dimension psychanalytique :

"La génétique des populations est une science qui a entre autres permis d'établir un arbre de filiation des populations humaines. A cet arbre de filiations correspondent les 300 à 400 familles de langues venues de la Langue Mère au fil des migrations ; Greenberg a, par ses travaux, réussi à mettre à jour toutes ces langues-filles. Chacune de ces familles évolua aussi [...], ces évolutions, couplées à des migrations finirent par donner naissance, petit à petit, et sur des siècles et des siècles, aux 6800 langues qui sont aujourd'hui parlées sur la Terre.

Personne ne sait ce que devinrent Nigiro et La Rousse. Tu sais en revanche, cher lecteur, que c'est grâce à leur histoire d'amour et à leur désir commun de ressembler à des hommes davantage qu'à des bêtes que tu peux aujourd'hui parler... étonnant non?"

Ainsi, de même que Gaston Bachelard, qui place le désir au cœur du phénomène de reproduction du feu par l'homme, Marie fait du sentiment amoureux le fond originel du langage articulé.

\section{Conclusion}

Dans le numéro 133-134 de Pratiques, Y. Reuter propose une typologie provisoire des fonctions didactiques du récit dans le cadre des apprentissages disciplinaires. Il distingue ainsi 7 fonctions :

- La fonction véhiculaire : « le récit sert de support, de médium, pour mettre en place, exposer, construire, évaluer des savoirs ou des savoir-faire [...] voire des compétences plus générales » (Reuter, 2007 : 9) dans et par le langage,

- Une fonction structurante : « le récit sert à établir des relations, organiser des contenus » en les insérant dans une trame narrative,

- La fonction objectivante : «le récit sert à actualiser, à incarner [...] en singularisant les genres, [...], en exemplifiant et en illustrant le cours [...] en objectivant au moins partiellement, ce qui est autrement invisible ou plus difficilement appréhendable (le raisonnement de l'élève, sa démarche de production »,

- La fonction médiatrice : «le récit sert, [...], de phase intermédiaire pour progresser vers une étape estimée cognitivement supérieure par l'école »,

- La fonction implicative : «le récit sert à " engager» l'élève dans le travail, à la sortir d'une position d'extériorité » (articulation cognition-affects via les personnages, lien avec le vécu personnel de l'élève) »,

- La fonction intégrative : " le récit sert non seulement à construire des normes, une vision du monde et de la discipline mais encore, [...] à intégrer les élèves $[. .$.$] dans des communautés déterminées et à constituer des sujets s'en-$ gageant dans des formes requises »,

- La fonction transitionnelle : « le récit sert de passerelle entre les disciplines, les mettant en relation et en interaction, les intégrant parfois aussi ».

L'expérience menée depuis deux ans nous amène à confirmer la pertinence de cette typologie. Au terme de cet article il nous faut cependant lui rajouter une nouvelle fonction : le récit relie 1'univers conceptuel à l'univers affectif, artistique, 
sensitif, onirique du sujet... Il participe à l'articulation entre « création graphique » et « raison graphique ». Il est à la fois générateur d'émotions, de sensations et d'idées. C'est pourquoi il doit trouver sa place et sa légitimité aux côtés des autres écrits utilisés dans la construction et l'évaluation des connaissances à l'université ${ }^{(7)}$. Car en mettant en mouvement la pensée il permet cette alchimie qui fait l'or des découvertes.

\section{Bibliographie}

Audiger, F., Ronveaux, C. (2007) : «Récit d'histoire, récit de fiction. Exemple de l'expérience de la guerre », Pratiques, $n^{\circ} 133-134$.

BaChelard, G. (1949, réédition 1986) : La psychanalyse du feu, Paris, Folio Essais.

Bautier, E., Manesse, D., Peterfalvi, B., Verin, A., (2000) : « Le cycle de vie du cerisier : une narration "scientifique" ?», in Repères $\mathrm{n}^{\circ} 21$, Diversité narrative, dir Y. Reuter, C. Tauveron, Paris, INRP, pp. 143-164.

BRUNER (2005) : Pourquoi nous racontons-nous des histoires? Le récit au fondement de la culture et de l'identité individuelle, Paris, Agora-Pocket.

Chabanne, J.-P., DunAs, A. (1999) : « La créativité “ordinaire”, Penser et apprendre, c'est créer les formes singulières de sa pensée et de son savoir », in Le français aujourd'hui, $\mathrm{n}^{\circ} 127$, pp. 17-24.

Chinier, J., Hass, G., Lorrot, D., Moreau, P., Mourey, J. (1990) : Les systèmes d'écriture. Un savoir sur le monde, un savoir sur la langue, Dijon, CNDP.

Delamotte, R., Gippet, F. Jorro, A., Penloup, M.-C., (2000) : Passages à l'écriture, Un défi pour les apprenants et les formateurs, Paris, PUF.

FRANÇOIS, F. (2006) : «Fiction-imagination-écrit-école : quelques remarques », in Repères $\mathrm{n}^{\circ} 33$, La fiction et son écriture, dir. S. Plane, F. François, Lyon, INRP, P. 197-221.

FRIER, C. (2004) : «Articulation théorie/pratique/implication personnelle dans le compte-rendu de stage en formation préprofessionnelle », in Actes du 21 ème congrès international de l'Association internationale de Pédagogie universitaire, 3-7 Mai 2004, Marrakech.

— (1998) : «Profils de lecteurs et modalités d'approche des textes de spécialité à l'université », in La didactique du français dans l'enseignement supérieur : Bricolage ou rénovation?, dir. Fintz, C., Paris, L'Harmattan, pp. 74-88.

LAFONT, J. (1999) : Pour une ethnolinguistique des ateliers d'écriture : analyse de pratiques sur plusieurs terrains. Thèse de doctorat, Université François Rabelais, Tours.

(7) Mais également nous semble-t-il à tous les niveaux de la scolarité et plus particulièrement dans le cadre de dispositifs pluridisciplinaires. 
KARA, M. (2004) : «Pratiques de la citation dans les mémoires de maîtrise », in Pratiques $\mathrm{n}^{\circ} 121-122$, Les écrits universitaires, Dir. Boch, F., Reuter, Y., pp. 111142.

NeUmayer, M., Neumayer, O., (2003) : Animer un atelier d'écriture, Faire de l'écriture un bien partagé, Paris, ESF.

Penloup, M.-C., dir. (2007) : Les connaissances ignorées. Approche pluridisciplinaire de ce que savent les élèves, Paris, INRP.

Petitjean, A. (2005) : «Ecriture d'invention au lycée et acquisition de savoir et de

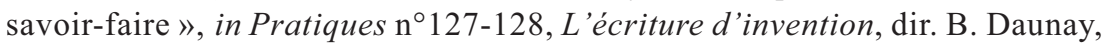
A. Petitjean, pp. 75-96.

Platon, Phèdre, http ://wikisource.org/wiki/phèdre

REUTER, (2007) : «Récits et disciplines scolaires. Présentation du numéro », in Pratiques n¹33-134 : Récits et disciplines scolaires, Dir. Reuter, Y., pp. 3-12.

RINCK, F. (2004) : «Construire la problématique d'un rapport de stage : les difficultés d'étudiants du second cycle », in Pratiques $\mathrm{n}^{\circ} 121-122$, Les écrits universitaires, Dir. Boch, F., Reuter, Y., pp. 93-110.

SAUZEAU, C., T RIQUET, E. (2004) : L'atelier d'écriture d'un récit de fiction scientifique: un dispositif interdisciplinaire pour un nouveau genre?, in Actes du $9^{\mathrm{e}}$ colloque de l'AIRDF, Québec.

WeINBERG, A. (2000) : «L'écriture, un tremplin pour la pensée », in Sciences Humaines $\mathrm{n}^{\circ} 190$, pp. 22-25.

\section{Annexe 1}

\section{Les critères d'évaluation de la fiction scientifique :}

1) Structure du récit : la fiction doit respecter les contraintes du récit ( $c f$. «structure narrative » du texte, cohérence textuelle), l'univers fictionnel ayant pour but de distraire le lecteur. (6 points)

2) Qualité de l'information scientifique : le récit doit comporter un point de vue sur une question scientifique, intégrant par conséquent des éléments à caractère scientifique ayant pour but d'instruire le lecteur. Ce critère (présence d'un contenu scientifique et d'un point de vue) implique une bonne compréhension des textes du dossier, de la précision (définitions, références à des auteurs, repères chronologiques...), de la rigueur (formulation des idées). (6 points)

3) Qualité de la formulation/expression : originalité, inventivité, poésie du texte, richesse du lexique, attention portée au « signifiant», humour, réinvestissement des « pépites de 1'atelier», etc. (6 points)

4) Présentation : soin apporté au document : typographie, illustrations... (2 points) 


\section{Annexe 2}

\section{Eléments de connaissance scientifique présents dans le dossier « Naissance et mutations de l'écriture»}

\begin{tabular}{|c|c|c|c|c|}
\hline Mots clés & $\begin{array}{l}\text { Concepts/ } \\
\text { notions/ } \\
\text { Définitions/ } \\
\text { mots clés }\end{array}$ & $\begin{array}{l}\text { Théories / } \\
\text { points de vue/ } \\
\text { approches }\end{array}$ & $\begin{array}{l}\text { Idées / } \\
\text { arguments }\end{array}$ & $\begin{array}{l}\text { Références } \\
\text { (Auteurs, dates, } \\
\text { lieux) }\end{array}$ \\
\hline $\begin{array}{l}\text { - Systèmes d'écriture } \\
\text { - Code écrit } \\
\text { - Fonctionnement } \\
\text { de l'écriture } \\
\text { - Origine de } \\
\text { l'écriture } \\
\text { - Ecriture et mémoire } \\
\text { - Ecriture et pensée } \\
\text { - Ecriture et } \\
\text { cognition } \\
\text { - raison graphique } \\
\text { - Littératie } \\
\text { - Communication } \\
\text { scripturale / orale }\end{array}$ & $\begin{array}{l}\text { - Signes pictographi- } \\
\text { ques / écriture des } \\
\text { choses } \\
\text { - Idéogrammes, pho- } \\
\text { nogrammes, cunéi- } \\
\text { formes, hiérogly- } \\
\text { phes, rébus à trans- } \\
\text { fert } \\
\text { - Principe acrophoni- } \\
\text { que } \\
\text { - Ecriture alphabéti- } \\
\text { que } \\
\text { - Phonèmes, graphè- } \\
\text { mes } \\
\text { - Code écrit } \\
\text { - Arbitraire du signe } \\
\text { - Signes symboliques } \\
\text { - Signes convention- } \\
\text { nels } \\
\\
\text { - Système de repré- } \\
\text { sentation des idées } \\
\text { - Raison graphique }\end{array}$ & $\begin{array}{l}\text { - Un système auto- } \\
\text { nome de représenta- } \\
\text { tion des idées } \\
\text { - Illusion évolution- } \\
\text { niste, « alphabéto- } \\
\text { centrisme " } \\
\text { - l'écriture constitue } \\
\text { une révolution intel- } \\
\text { lectuelle, un bond } \\
\text { dans l'histoire de la } \\
\text { pensée } \\
\end{array}$ & $\begin{array}{l}\text { - l'écriture est le } \\
\text { moyen le plus sim- } \\
\text { ple et le plus efficace } \\
\text { pour transcrire la } \\
\text { parole } \\
\\
\text { - L'écriture est un } \\
\text { piège : elle n'est que } \\
\text { l'illusion de la con- } \\
\text { naissance et de la } \\
\text { science } \\
\text { - l'écriture possède } \\
\text { une indépendance } \\
\text { vis-à-vis de la parole }\end{array}$ & $\begin{array}{l}\text { - Sumer, } 3500 \text { av. JC } \\
\text { - Egypte, } 3200 \text { av. JC } \\
\text { - Phénicie, } 1200 \text { av. JC } \\
\text { - Grèce : } 650 \text { av. JC } \\
\text { - J.J. Glassner (2000) }\end{array}$ \\
\hline
\end{tabular}

